\title{
Efficient Energy Management for Target Tracking in Wireless Sensor Network
}

\author{
Dong-Mei Yan*, De-Ying Gu, Bin Wang \\ Control Engineering Department, Northeastern University at Qinhuangdao, China No.143, Taishan \\ Road, Qinhuangdao, Hebei, China \\ dong_117@126.com \\ ${ }^{*}$ Corresponding author
}

Keywords: Energy management, Wireless sensor network, Target tracking.

\begin{abstract}
A Wireless Sensor Network consists of many sensor nodes built in sensing, data processing, and communicating components, which has found its wide use in health, military, and security. Each node with limited energy supports sensor operations, on-board signal processing, and communication with neighboring nodes. So, Power conservation is a challenging accomplishment for wireless sensor network because each sensor node's power recourses are limited. How to extend the lifetime of the network should be carefully considered in designing wireless sensor network. In this paper we proposed an energy efficient dynamic energy management technique for target tracking in Wireless Sensor Network, We utilize PSO algorithm and duty cycling technology in order to let sensors that perform tasks be in the active state and all other sensors are in the sleep state. The proposed mouthed is useful for manage sensor nodes to extend network lifetime.
\end{abstract}

\section{Introduction}

Wireless sensor network (WSN) is one of the most important technologies in the 21 st century. WSN which is composed of large amount of sensor nodes that are embedded onboard kinds of sensors, such as infrared sensor, magnetic modes, acoustic sensor, seismic censor, imagers and so on. Neighboring nodes are linked by wireless mode, whose position can be located by GPS or positioning algorithms. WSN has found wide applications in military sensing, distributed robotics, industrial and manufacturing automation, environment monitoring, and building monitoring.

Sensor nodes with limited energy must supports sensor operations, on-board signal processing, and communication with neighboring nodes. So it is important to design power-efficient signal processing algorithms and efficient communication protocols [1].

The lifetime of a sensor network is decided by the power consumed at each sensor node. Thus, a efficient energy management can provide a longer network lifetime. Several methodologies have been proposed to design energy efficient communication process, sensor node operating system and sensor node circuits. A variety of DPM (Dynamic Voltage Management) techniques have also been proposed to reduce the energy $r$ consumption in sensor nodes. Much work has been done to research sleep state and active power management, DVS (Dynamic Voltage Scaling), battery state awareness power management and so on.

The main goal of energy management is to prolong the lifetime of WSN because it is impossible to replace battery once the sensor network has been. So, power conservation technique mused be exploited in order to save the energy [2].

In this paper we proposed an energy efficient dynamic energy management technique for target tracking in Wireless Sensor Network, We utilize dynamic cluster and duty cycling technology in order to let sensors that perform tasks be in the active state and all other sensors are in the sleep state. The proposed mouthed is useful for manage sensor nodes to extend network lifetime.

\section{Energy Management of Sensor Node}

\section{Radio Energy Dissipation Model of Sensor Node}

The radio hardware energy dissipation model is shown in Fig. 1, which assumed an $r_{2}$ energy loss due to channel transmission. So, to transmit $k$-bit message a distance $d$ employing the radio model, the radio expends [3]. 


$$
E_{T x}(k, d)=E_{T x-\text { elec }} \times k+\varepsilon_{\text {amp }} \times k \times d^{2}
$$

And to receive this message, the radio expends

$$
E_{R x}(k)=E_{\text {elec }} \times k
$$

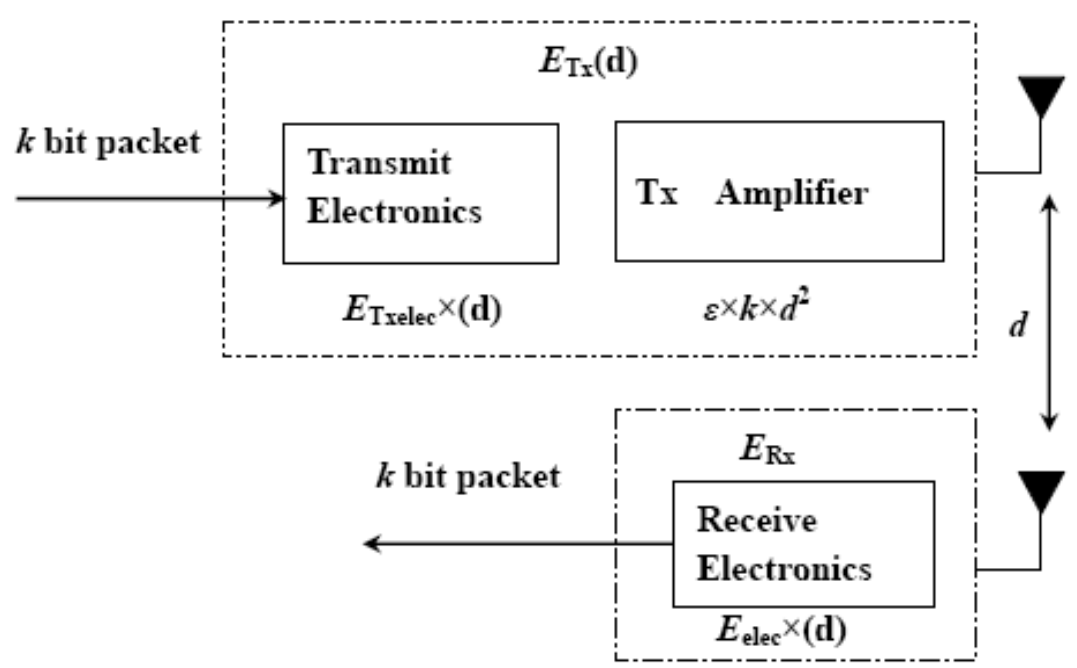

Fig.1 Radio model

\section{Proposed Relative Technology of Energy management}

Energy management is an important means to save energy. The goal of energy management is to reduce energy consumption and balance energy among all the nodes in wireless sensor node. Many kinds of methods for energy management are proposed including node energy management, MAC protocols, routing protocols, cross-layer optimization and so on [4].

MAC protocol. MAC Protocol in WSNs is the bottom part of the network communication protocol, which is mainly used to set up rules for the nodes to share digital media. Compared to traditional networks, energy efficiency is a primary consideration in the design of MAC protocol of WSNs network. Some reasons of energy waste in WSNs have been concluded by [5]. They are idle listening, message collision, overhearing, control-packet overhead and over-emitting and so on.

Researchers have developed a variety of different MAC protocols for different applications of wireless sensor networks. The challenge in designing a MAC protocol is to make a balance between energy conversation and flexibility of the network. Apparently, it is always easier to achieve a better performance with higher energy consumption. It is a challenging problem to balance the energy conversation and the performance of wireless sensor networks.

Node Energy Management. The main objective for a single node in wireless sensor networks is to make itself work permanently. Vinod Sharma etc. proposed an optimal energy management policy for energy harvesting sensor nodes, in which the generated energy should be stored in a buffer. Then the sensor node in wireless sensor networks could detect a random field and generates a packet periodically, which is stored in a queue and transmitted using the energy available at that time [6]. Pering $\mathrm{T}$ etc. proposed a new concept named dynamic voltage scaling (DVS) [7], which means it could dynamically adjusts the supply voltage of the microprocessor according to the system load. DVS has a perfect effect on energy conservation, but system must have ability to measure or predict the system load.

Cross-layer optimization. Generally, most communication protocols of sensor networks are designed with hierarchical structure that is called layers independent of each other, and layers are relatively simple. So networks with different systems are easy to communicate. However, cross-layer optimizations is proposed to enhance the information communication among network layers in order to achieve a better efficiency with optimized system performance, so layers can avoid being interfered each other. 
Routing protocol. Main objective of routing protocol of wireless sensor network is to establish best path between source nodes and sink nodes. At the same time, the routing protocol should also have some characteristics such as energy saving, fault tolerance and low latency. And energy saving is definitely the main principle in routing protocol design.

Energy routing protocol is one of routing protocols for wireless sensor networks firstly proposed. The global information of the whole network is needed for routing. However, due to energy constraints in wireless sensor networks, the node can only access the topology information of local network.

Each kind of routing protocol has its own advantages and disadvantages, the selection of the routing protocol should depend on network environment.

\section{Efficient Energy Management for Target Tracking}

\section{Consumption of Nodes.}

Each sensor node with limited energy $(<0.5 \mathrm{Ah}, 1.2 \mathrm{~V})$ contains four components: a sensing unit, a processing unit, a transceiver unit and a power unit. Moreover, it may be impossible to replace power resources in special environment. Thus, Power management of sensor nodes is very important for wireless sensor network[8].

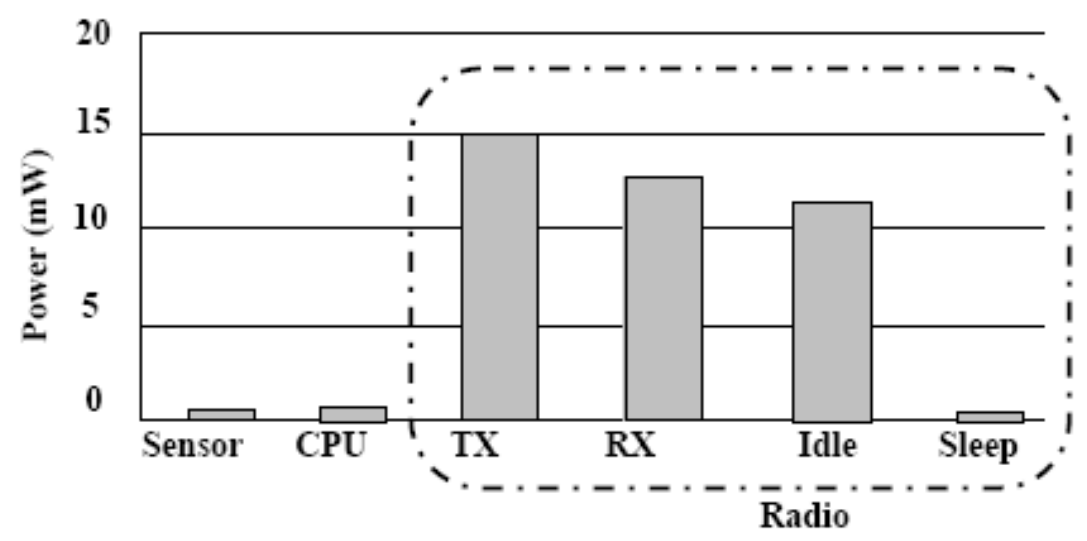

Fig .2 Energy Consumption of Nodes

Energy consumption of node subsystems is shown in Fig.2 . There are four possible states of sensor node's radio: transmit (TX), receive (RX), idle, or sleep. Obviously, it is obtained from the Fig.2

$$
E_{T X} \approx E_{R X} \approx E_{I D L E}>>E_{\text {Sleep }}
$$

Thus, Sensor nodes should be completely shut down the radio when they are not transmitting or receiving data.

\section{Efficient Energy Management Using PSO}

PSO Algorithm. Several scientists have achieved computer simulations of the movement in a bird flock or fish school. These scientists considered that local process might base on the uncertain group dynamics of bird social behavior. Models built by those scientists depended heavily on control of inter-individual distances. This means that the synchrony of flocking behavior can be expressed by a function which described birds' efforts to maintain an optimum distance between themselves and their neighbors.Particle swarm optimization is a very useful algorithm for optimizing a wide range of functions, which is highly dependent on stochastic processes .

Duty cycling. Duty cycling is very important in wireless sensor for con-serving energy, which makes the radio transceiver be in the sleep mode while communication is not required. Thus, sensor nodes change between active and sleep state based on network activity, which is known as duty cycling. Duty is cycling defined as time slots that nodes are active during their lifetime. In target tracking, since sensor nodes per-form a task cooperatively, it is necessary to coordinate their sleep/wakeup times. A 
sleep/wakeup scheduling algorithm is used to decide state transformation (active to sleep and vice versa) of sensor nodes.

Energy Optimization. Energy consumption of a wireless sensor node consists of two main parts: Energy for transmitting, receiving and amplifying data and energy for processing data so total energy loss E of a sensor node:

$$
E=E_{T X}+E_{R X}+E_{\text {sup }}+E_{\text {leak }}
$$

While Esup is related to the supply voltage, Eleak is decided by leakage current.

As mentioned above, $E_{T X}+E_{R X}=\left(2 E_{\text {elec }}+\varepsilon_{\text {amp }} d^{2}\right) b$, so, $E_{T X}+E_{R X} \gg E_{\text {sup }}+E_{\text {leak }}$

Provided that there are $m$ set of clusters and $n_{\mathrm{j}}$ sensors in the cluster $C_{\mathrm{j}}$, where $1<\mathrm{j}<=\mathrm{m}$. Assume a sensor $S_{\mathrm{ij}}$ in the cluster $C_{\mathrm{j}}$ is at distance $d_{\mathrm{ij}}$ to the cluster head $C H_{\mathrm{j}}$, and $C H_{\mathrm{j}}$ is at a distance of $D_{\mathrm{j}}$ to the sink. The energy consumption of all the sensors and clusters can be expressed by [10]:

$$
E \approx \sum_{j=1}^{m} \sum_{i=1}^{n}\left(d_{i j}^{2}+\frac{D_{j}^{2}}{n_{j}}\right)
$$

Then the fitness function is given by:

$$
\text { Fitness } E=\min \sum_{j=1}^{m} \sum_{i=1}^{n}\left(d_{i j}^{2}+\frac{D_{j}^{2}}{n_{j}}\right)
$$

\section{Sensor Management During Target Tracing to save enegy}

Predication of trajectory. When to wakeup sensor nodes for tracking target is mainly based on the location of target, so it is very important to estimate the trajectory of targets during target moving. Kalman filter is utilized to predict the trajectories of target in this paper. Equations for the Kalman filter include time update equations and measurement update equations. The time update equations represent projecting forward the current state and error covariance estimates to achieve the a priori estimates for the next time step.

The state vectors of all targets at time tk is expressed by $x_{k}=\left[x_{k}^{1}, x_{k}^{2}, \ldots, x_{k}^{n}\right]$, where $x_{k}^{i}$ is the state vector of target $\mathrm{i}$ and $\mathrm{n}$ represents the number of targets. The state equation of the $\mathrm{n}$ targets is set by

$$
X_{k}=A \cdot X_{k-1}+W_{k-1}
$$

with a measurement that is

$$
Z_{k}=H_{k}+V_{k}
$$

$W_{\mathrm{k}}$--process and measurement noise

$V_{\mathrm{k}}$-- measurement noise (respectively).

A -- a linear function

Time update equations and measurement update equations is expressed by [11]

$$
\begin{aligned}
& X(k \mid k-1)=A \cdot X(k-1 \mid k-1)+W(k) \\
& P(k \mid k-1)=A \cdot P(k-1 \mid k-1) A^{T}+Q
\end{aligned}
$$




$$
\begin{aligned}
& X(k \mid k)=X(k \mid k-1)+K_{g}(k)(Z(k)-H \cdot X(k \mid k-1)) \\
& K_{g}=P(k \mid k-1) \cdot H^{\prime} /\left(H \cdot P(k \mid k-1) H^{T}+R\right) \\
& P(k \mid k)=\left(I-K_{g}(k) \cdot H\right) P(K \mid k-1)
\end{aligned}
$$

The process is repeated with the previous a posteriori estimates used to predict the new a priori estimates with each time and measurement update.

When a target moves into the area of sensor network, sensor nodes are selected to form a cluster dynamically, and then the sensor's information is transmitted to cluster heads. Different sensor nodes are chosen to form another cluster with the target moving, and the nodes in this cluster are awakened while other sensor nodes can be made into sleep mode based on the prediction of trajectory of target and nodes' energy, so energy of whole network is balanced and the lifetime is prolonged effectively.

\section{Summary}

In this paper, we propose an Efficient Energy Management for Target Tracking in Wireless Sensor Network. We utilize PSO algorithm and duty cycling technology in order to let sensors that perform tasks be in the active state and all other sensors are in the sleep state. The proposed mouthed is useful for manage sensor nodes to extend network lifetime.

\section{Acknowledgment}

This work was supported by the National Natural Science Foundation of China (No. 61403067), the Natural Science Foundation of Hebei Province (No. F2013501075) and the Doctoral Scientific Research Foundation of Liaoning Province (No. 20131030).

\section{References}

[1] Chee-Yee Chong; Kumar, S.P.,Sensor Networks: Evolution, Opportunities, and Challenges, Proceedings of the IEEE, 91 (2003) 1247-1256.

[2] Shelke, R.; Kulkarni, G.; Sutar, R.; Bhore, P.; Nilesh, D.; Belsare, S,.Energy Management in Wireless Sensor Network, 15th International Conference on Computer Modelling and Simulation, (2013) 668-671.

[3] W.R. Heinzelman,. A. Chandrakasan and H. Balakrishnan, Energy-Efficient Communication Protocol for Wireless Microsensor, Networks, The 33rd Annual Hawaii International Conference on System Sciences, Hawaii, Island of Maui, USA, (2000):1-10.

[4] Wan, Z.G.; Tan, Y.K.; Yuen, C. Review on energy harvesting and energy management for sustainable wireless sensor networks, 2011 IEEE 13th International Conference on Communication Technology (ICCT), (2011) 362-367.

[5] ZHENG Guo-Qiang, LI Jian-Dong, ZHOU Zhi-Li. Overview of MAC Protocols in Wireless Sensor Networks". ACTA AUMATICA SINICA, 34(3), (2008) 305-316.

[6] Vinod Sharma, Utpal Mukherji, Vinay Joseph. Optimal Energy Management Policies for Energy Harvesting Sensor Nodes, IEEE Transactions on Wireless Communications, vol. 9, no. 4, (2010) 1326-1336. 
[7] PeringT, Burd T, Brodersen R. "Dynamic voltage scaling and the design of a low-power microprocessor system". Proceedings of Power Driven Micro architecture Workshop, attached to ISCA 98. Barcelona, Spain, (1998) 107-112.

[8] D. Estrin, Wireless sensor networks tutorial part IV: sensor network protocols, Mobicom 2002, USA, (2002) IV1-IV60.

[9] J. Kennedy and R. Eberhart, Particle swarm optimization, IEEE International Conference on Neural Networks, Perth, WA, ustralia, (1995) 1942-1948.

[10] Y. Shi and R. C. Eberhart, A modified particle swarms optimizer, The IEEE International Conference on Evolutionary Computation Proceedings, IEEE World Congress on Computational Intelligence, Anchorage, Alaska, USA, (1998) 69-73.

[11] Y. Shi and R. C. Eberhart, Empirical study of particle swarm optimization, Proceedings of the 1999 Congress on Evolutionary Computation,, Mayflower Hotel, Washington, D.C., USA, (1999) 1945-1950,.

[12] Feng Zhao; Jie Liu; Juan Liu; Guibas, L. and Reich, J., Collaborative signal and information processing: an information-directed approach, Proceedings of the IEEE, vol.91, (2003)1199-1209.

[13] Guru, S.M.; Halgamuge, S.K. and Fernando, S., Particle Swarm Optimisers for Cluster formation in Wireless Sensor Networks, 2005 International Conference on Intelligent Sensors, Sensor Networks and Information Processing Conference, Melbourne, Australia, pp:319-324, 2005.

[14] Greg Welch and Gary Bishop, An Introduction to the Kalman Filter, TR 95-041, Department of Computer Science University of North Carolina at Chapel Hill.

[15] Dongmei Yan, Jinkuan Wang, Li Liu and Bin wang, Sensor Scheduling Target Tracking-oriented based on Particle Swarm Optimization, The 30th Chinese Control Conference, (2011) 5050-5053.

[16] Dongmei Yan, Jinkuan Wang, Sensor Scheduling Algorithm Target Tracking-oriented, Wireless Sensor Network, vol.3, no.8, (2011) 295-299.

[17] Dongmei Yan; Jinkuan Wang; Sensor Management for Target Tracking in Wireless Sensor Network , ICIC Express Letters, Part B: Applications, vol.3, No.2,(2012) 291-296. 